\title{
DENSITY OF STATES OF AMORPHOUS HYDROGENATED SI
}

\author{
P. Lemaire* and J.P. Gaspard \\ Université de Liège, Institut de Physique, B 4000 Sart-Tilman, Belgium \\ * and IRSIA, Brussels, Belgizon
}

\begin{abstract}
In this paper, we analyze the electronic density of state of amorphous semiconductors with a derigling bond, elther unsatisfied or satisfied hy a monovalent atom (H. Cl. F ... a as it is now currently achieved. The electronic properties are calculated within the tight binding framework. In the simplified case of the Eethe lattice, exact formulas are obtained. We observe an isolated dangling fond state in the gap. with a weight depending on the gap wioth. The density of states is also obtained when an ajatom is covalently attached to the dangling bond. The range of parameters required for emptying the gap is analyzed. Finally more sophisticated numerical calculations are done on a realistic hand uvilt soo atom model.
\end{abstract}

1. Introduction

It is well established that amorphous group IV semiconductors have had electrical properties because their gap has a too large density of states; in particular they cannot be doped. The presence of bonded hydrogen reduces the density of
localized states in the mobility gap to values less than $10^{10} \mathrm{~cm}^{-3}$ $\mathrm{eV}^{-1}$ and $1 \mathrm{~s}$ essential for doping as demonstrated by spear and coworkers /1/.

The aim of this paper 15 the analysis of the electronic density of states (d.0.s.) of non ideal amorphous semiconductors i.e. With an isolated or a double dangling bond (d.b.). We also consider the case where the d.h. is satisfied by a monovalent atom e.g. H.

A tight hinding or LCAO formalism is used: it involves the 3 s and $3 p$ valence electrons of Si. Such a simple description is particularly useful in the case of strongly disordered system where more sophisticated band structure calculations cannot be appiled. The tight binding interaction parameters are obtained from more sophisticated band structure calculations on crystalline structures (e.g. see the values an Fig. 3). The valence band is then correctly reproduced but the conduction band - corresponding to extended electronlc states - is noorly described.

The poper is divided into two sections: in section 2 exact results are obtained in some schematic situations. In section 3 the local density of states is calculated on various orbitals of a hand bullt structural model and the results are discussed in term of the experimental data. 
2. Exact results for the isolated, double and hydrogenated dangling Bond

It is well known that the local density of states in tight binding systems depends primarily on the local environment of the atoms (see also section 3 ). Consequentiy a model that describes correctly the local coordination and position of the atoms gives the trends of the electronjc properties, at least in the valence band and the gap region.

A Bethe lattice (B.1.) 1s a structure with a given coordination $N$ everywhere but with no closed rings of atoms $/ 2 /$. The advantage of the Bethe lattice is that exact formulas can be simply obtained and they allow a rapid analysis when varying the parameters of the problem. It is closer to reality than the molecular modeli the valence and conduction hand have a finite bandwidth in the B.l. approximation whereas in the molecular model the bands are reduced to energy levels. Thus the $B, 1$. allows a discussion of the absorption of gap states in the bands e.g. as shown on Fig. 1. However the E. 1. bandwidth suffers from a narrowing of the band due to the ahsence of closed rings. As the shortest rings are sixfold in the diamond lattjoe (fivefold $1 n$ the amorphous structurel, the B.l. approximation is more accurate in open structures than in compact phases (FCC ...) where threefold rings are present.

In a simple tight binding description (usually referred to as the Weaire-Thorpe hamiltonian/3/J with only two resonance (or hopping) integrals: B (interatomic) hetween two orbitals pointing in the direction of the hond and $\varepsilon\left(=\left(F_{-}-F_{s}\right) / N\right.$, tntraatomic) between two equivalent son-g orhitals.on.the same atom of coordination $N$, the local Green function (resolvant) on the dangling bond takes the simple analytic form

$$
G_{d \theta}(z)=\frac{1}{z-\frac{(N-1) \varepsilon^{2}}{z-(N-2) \varepsilon-B^{2} G_{d b}(z)}}
$$

It is easily demonstrated that a dangling bond peak appears in the gap at the energy $E_{0}=\left(E_{s}+(N-1) F_{p}\right) / N$, Indepen dantly of the $B$ parameter, with a welght

$$
W \quad=1-(N-1) \varepsilon^{2}, B^{2}
$$

for $B \varepsilon>V N-1$, i.e. In the semiconducting region. Let us notice in addition that as the dangling bond state falls above the fermi level, charge trarsfer effects should lower the dangling bond level, except in the case of the linear chatn $(N=2)$.

Whether or not a double dangling fond exists in a-si is an open question: at least the existence of $\mathrm{SiH}_{2}$ has heen proved by infrared spectroscopy. The electronic states of a double $\operatorname{sp}^{3}$ d.b. differ qualitatively from the jsolated d,b. A state in the gap moves from $\varepsilon$ to $-\varepsilon$ (Fig. 1) with a weight increasing like (2). A second non resonant localized state appears at $E_{p}$ with the constant weight 0.5 . The Green function writes:

$$
G_{2 d b}(z)=\frac{1}{2} \frac{1}{z-\varepsilon}+\frac{1}{2} \frac{1}{z-\varepsilon-\frac{2 \varepsilon^{2}}{z-\beta^{2} G_{d b}(z)}}
$$




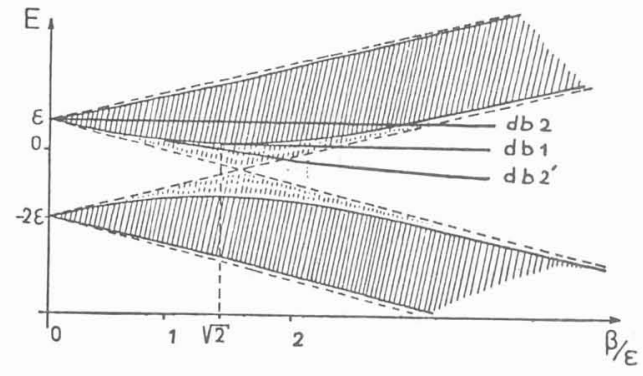

Fig. 1 - Position of the bands and dangling bond states.

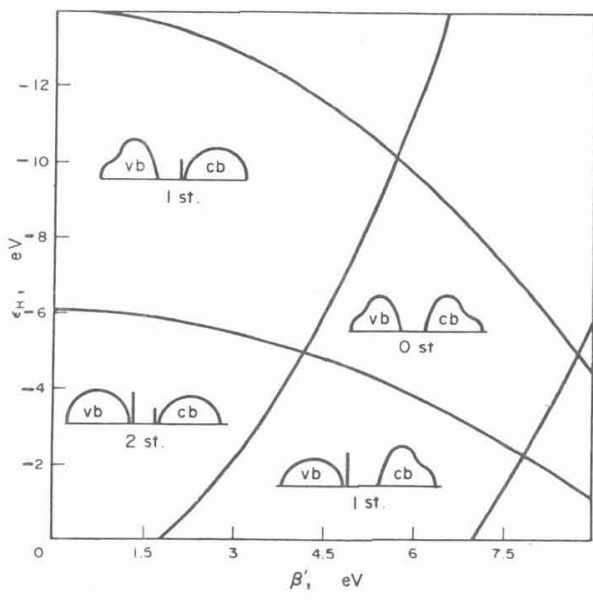

fig, 2 - Variation of the number of localized states in the gap. /4/

It is well established that the presence of bonded hydrogen (or other elements) in a-si is essential for the electrical and doping properties. It is easy to write down a general formula relating the Green function on $H$ to the d.b. Green function

$$
E_{H}(z)=\frac{1}{z-\varepsilon_{H}-B^{2} G_{d b}(z)}
$$

where $B^{\prime 2}$ is the effective interaction Between $H$ is and the dangling orbital of $\mathrm{Si}$. When varying $\varepsilon_{H}$ and $B$, one observes the existence of 0,1 or 2 states in the gap. Fig. 2 shows which yalues of $\varepsilon_{H}$ and $\beta$ are necessary for emptying the gap, Dna soвs that for physical values of $\beta(3-4$ ev) the d.b. state enters the conduction band $/ 4 /$.

\section{Density of $s p$ states on a realistic structure}

We have ouilt a realistic model of amorphous fourfold coordinated $S i$ containing 500 atoms around an isolated dangling bond. Seven complete shelis of nearest neighoours are developped around tha central atom. We have checked that the palr correlation function $L_{1}=2.35 \mathrm{~A}^{0}, \sigma_{\eta}=0.06 \mathrm{~A}^{0}, r_{2}=3.82 \mathrm{AO}$. $\left.\sigma=0.21 \mathrm{~A}^{0}\right)$ and the angular distribution $\left(\theta^{2}=309,19^{\circ}\right.$,

$\sigma=8.54^{\circ}$, fit the experimental data on a-Si.

A tight binding hamiltonian is used to determine the electronic properties of the system: nearest neighbour interactions are taken from Chadi et al, $15 / 3$ the values are given on F1g. 4. As no relaxation of the model was performed, we ignored the variation of the parameters with interatomic distance. The absence of translational invariance requires the use of special techniques of calculation of the d.o.s.. described elsewhere /6/. The first 28 moments of the d.0.3. (twice the number of shells) are computed; this corresponds. to an energy resolution of the spectrum of the order of $y$. Fig. 4 shows the local d,o.s, on various orbitals of the atom bearing the d.b, as well as the average nsp(e). The d,B, points in the $z$ direction and the backbonds are three caplanar sp2 hybrids. The local d.o.s. n (E) on the p, (unsatiofled) orbital shows a narrow peak at $E_{p}$. $P^{z}$. Just below the conduction Band edge, as expected from the local geometry. As we learned from the B.1. results, a $s p^{3}$ type dangling Dand had given a peak at the lower energy $\left(E_{s}+3 E_{g}\right) / 4$. 

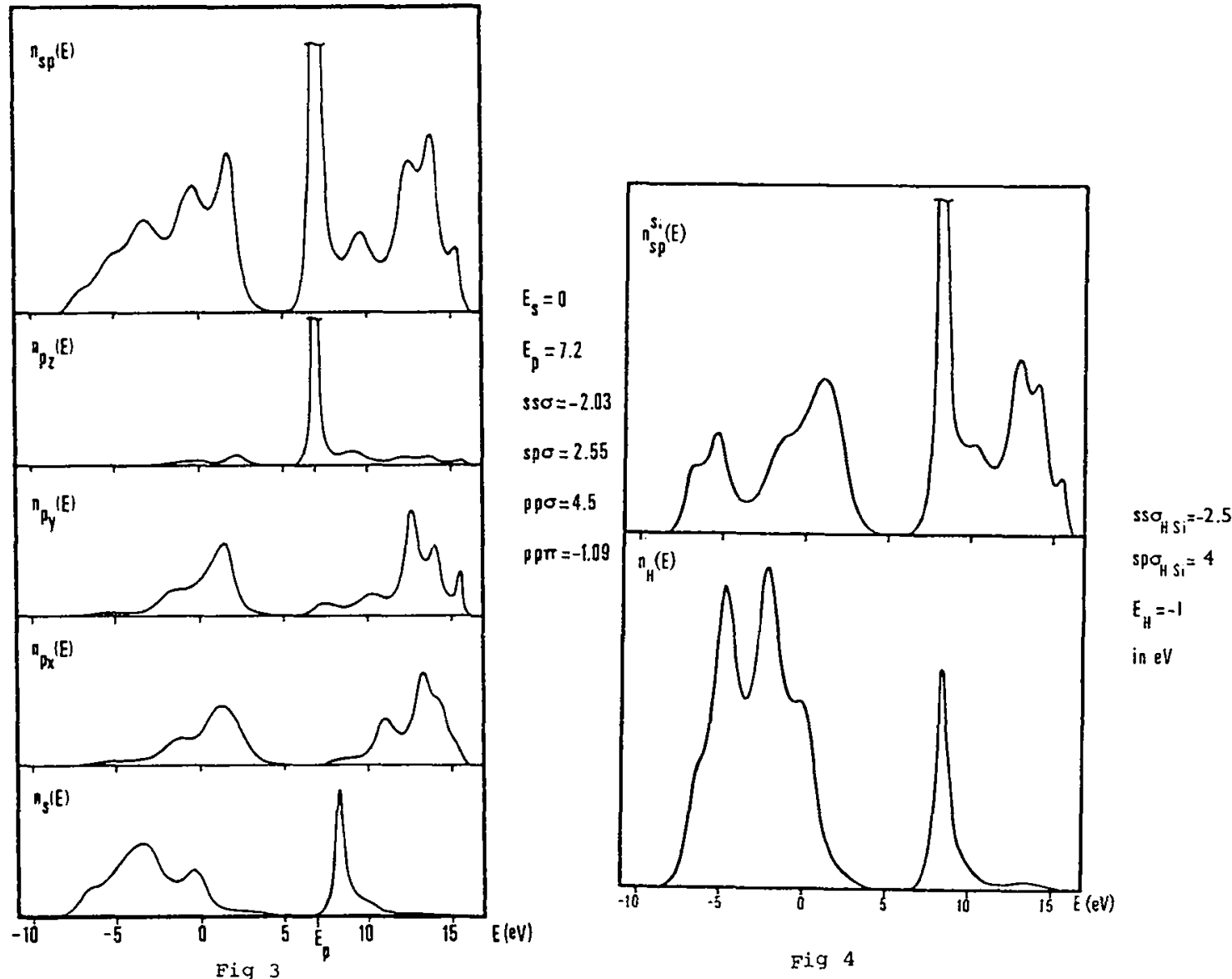

$\operatorname{Fig} 4$

The $n_{x}(e)$ and $n_{y}(e)$ densities of states are very similar to the corresponding $p$ density of states in fourfold coordinated system (crystaliline or amorphous). They show that the $p$ character of the states increases with increasing energy in the valence and the conduction bonds. By contrast, the bottam of the bonds are $s$ type as shown on $n_{s}(E)$, The top curve of Fig. 3 is the average of the curves drawr below: lit is characterized hy a reduction of the peok at the top of the valence band (relatively to the crystall and the appearence of the d.h. peak below the conduction bend edge.

The hydrogenation of the d.h. Cor its passivation by any other element) has a definite effect on the gap states. For the values of the H-Si interaction parameters mentioned an $F 1 g$. , the $p$ dangling bond states are pushed in the conduction band. In addition, the locald.o,s, on the $H$ atom show a three peak structure, two of them are at $-5 \mathrm{eV}$ and $-2.5 \mathrm{eV}$ in the valence oand. They can be related to the states observed in photoemission experiments $/ 7 /$. A smaller value of the interaction parameter (e.g. ss $\sigma=-1.5 \mathrm{eV}$, sp $\sigma=2.5 \mathrm{eV}$ ) produces neither the emptying of the gap nor the splitting of the $H$ peak in the valence band.

References

$/ 1 / W$. SPEAR and P.G. LE COMBER, Ph1l. Mag. 33 (1976) 935

$12 / \mathrm{J} . \mathrm{O}$. JOANNOPOULOS and M.L. COHEN, Solid State Phys.31 (1976)71
$13 / \mathrm{M}$. F. THORPE and D. WEAIRE; Phys. Rev.B4 (1971) 3518

$14 / \mathrm{P}$. LEMAIRE and J.P. GASPARO. Solid State Comm., 38 (1981) 397

/5/ D.J. CHADI and M.L. COHEN, Phys. State Sol. (b) 68 (1975) 405

$/ 6 / \mathrm{J} . \mathrm{P}$. GASPARD and F. CYROT-LACKMANN, J.Phys.C 6 (1973) 3077

/7/ B. VON RDEDERN, L. LEY, M, CARDONA and F.W. SMITH, PhI1.

Mag. B 40 (1979) 433 . 